\title{
Paragonimus westermani
}

National Cancer Institute

\section{Source}

National Cancer Institute. Paragonimus westermani. NCI Thesaurus. Code C123538.

A species of parasitic flatworms in the family Trog lotrematidae. The lifecycle of P. westermani involves freshwater snails as the first intermediate host, freshwater crustaceans as the second intermediate host, and humans or cats as the definitive host. Infestation causes paragonimiasis. 